\title{
PERSISTÊNCIA DE LINURON E ORIZALINA EM LATOSSOLO ROXO SOB CULTIVO DE SOJA $\left(^{1}\right)$
}

\author{
MARIA DO CARMO DE SALVO SOARES NOVO $\left({ }^{2}\right)$, LUCIANO SOUZA PAES CRUZ $\left({ }^{3}\right)$, \\ ELI SIDNEY LOPES $(2,6$, VIOLETA NAGAI $(4)$, LUIS ALBERTO AMBRÓSIO $(4)$, JOSÉ CARLOS \\ VILA NOVA ALVES PEREIRA $\left({ }^{5}\right)$ e MARIA LUIZA COLOGNESE DE OLIVEIRA LOMBARDI $\left({ }^{2}\right)$
}

\begin{abstract}
RESUMO
O ensaio foi efetuado durante dois anos consecutivos na Estaçăo Experimental de Ribeirão Preto, em latossolo roxo cultivado com soja IAC-11. O experimento foi em parcelas subdivididas com os tratamentos das parcelas principais dispostos em blocos ao acaso, com quatro repetiçós, sendo estudados os tratamentos seguintes: linuron, nas doses 1,00 e $2,00 \mathrm{~kg} / \mathrm{ha}$; orizalina, nas doses 1,875 e $3,75 \mathrm{~kg} / \mathrm{ha}$ e duas testemunhas sem herbicidas; nas subparcelas, estudaram-se as épocas de amostragem. As sementes de todas as parcelas tratadas com herbicida e uma das testemunhas foram inoculadas com Bradyrhizobium japonicum estirpe SMS-443 (= 29W). Foi feita a comparação quanto à sensibilidade dos bioensaios usando os métodos de Parker e Santelman. Verificou-se que, para a detecção de orizalina, o método de Parker foi o mais sensível, ocorrendo o inverso para o linuron. Em condiçőes de campo, a perda da atividade da orizalina, nas doses de 1,875 e $3,75 \mathrm{~kg} / \mathrm{ha}$, foi lenta, tendo persistido no primeiro ano até 112 dias após a aplicaçăo, enquanto, no segundo ano, as mesmas doses ainda reduziam $50 \%$ o crescimento da planta-teste até 84 e 145 dias respectivamente. Neste segundo ensaio, o final da persisténcia no solo para ambas as doses de orizalina ocorreu aos 390 dias da aplicação. Quanto ao linuron, embora sua fitotoxicidade inicial fosse bastante elevada, no primeiro ano, aos 42 dias, não havia mais sintoma de fitotoxicidade devido a qualquer uma das doses. No segundo ano, na aplicação de $1,00 \mathrm{~kg} / \mathrm{ha}$, aos 28 dias, 0 produto havia-se degradado em nível não detectado pela planta-teste, o que ocorreu para a dose de $2,00 \mathrm{~kg} / \mathrm{ha}$ somente aos 70 dias.
\end{abstract}

Termos de indexaçăo: soja, herbicida, orizalina, linuron, persistência.

\section{ABSTRACT \\ PERSISTENCE OF LINURON AND ORYZALIN IN A DUSKY RED LATOSOL CULTIVATED WITH SOYBEAN}

The trial was carried out during two sequential years in Ribeirão Preto Experimental Station, State of São Paulo, Brazil, in a Dusky Red Latosol cultivated with soybean IAC-11. Using a split-plot design; two dosages of linuron (1.00 and $2.00 \mathrm{~kg} / \mathrm{ha}$ ) and oryzalin (1.875 and $3.75 \mathrm{~kg} / \mathrm{ha})$ were compared with two control treatments: one inoculated with Bradyrhizobium japonicum strain SMS$443(=29 \mathrm{~W})$, and the other without inoculation. The experimental design was a randomized complete block-design with four replicates. The subplots were sampled on different days. The sensibility of Parker's and Santelman's methods for lab biossay was compared. The Parker's method performed more responsible for oryzalin while Santelman's for linuron. Under field conditions, the loss of activity of oryzalin was slow, persisting in the first year up to 56 and 84 days, for 1.875 and 3.75 $\mathrm{kg} / \mathrm{ha}$, respectively. In the second year, these dosages still decreased the plant-test growth in more than $50 \%$ at 84 and 145 days, respectively. Only after 390 days post applications oryzalin stopped

(1) Trabalho financiado pela EMBRAPA e, parcialmente, pela FAPESP. Apresentado na IV Conferencia Mundial de Investigación en Soja, Buenos Aires, em 5-9 de março de 1988. Recebido para publicação em 10 de dezembro de 1991 e aceito em 29 de setembro de 1992.

( ${ }^{2}$ Seção de Microbiologia do Solo, Instituto Agronômico (IAC), Caixa Postal 28, 13001-970 Campinas (SP).

(3) Seção de Fisiologia (IAC).

() Seção de Técnica Experimental e Cálculo (IAC).

(5) Estaçāo Experimental đe Ribeirão Preto (IAC).

6) Com bolsa de pesquisa do CNPq. 
affecting the plant growth. Although the initial phytotoxicity of linuron was very high, in the first year, there were no phytotoxicity symptom at $\mathbf{4 5}$ days in both dosages. In the second year, linuron was not detected at 28 days for the dosage of $1.0 \mathrm{~kg} / \mathrm{ha}$ and at 70 days for the dosage of $2.0 \mathrm{~kg} / \mathrm{ha}$.

Index terms: soybeans; herbicides, oryzalin, linuron, persistence.

\section{INTRODUÇÃO}

A preocupação com os efeitos dos herbicidas sobre a atividade microbiológica nos cultivos subseqüentes, em geral, está ligada à ausência de seletividade $\mathrm{e}$ à possibilidade de persistência no solo por período além do desejável para o controle eficiente das plantas daninhas.

A suscetibilidade da cultura à competição e o aumento considerável da área cultivada com soja no País, na última década, contribuíram para elevar o consumo de herbicidas nas lavouras dessa leguminosa. O linuron e a orizalina são dois produtos de classes químicas diferentes, recomendados para o controle de plantas daninhas em pré-emergência na cultura da soja (Hertwig, 1983).

No caso das leguminosas, há um interesse especial no estudo do efeito dos herbicidas, dada a possibilidade de influenciarem especificamente $o$ processo de fixação biológica do nitrogênio ou a nodulaçāo, afetando os rizóbios antes da formação dos nódulos.

Uma vez determinados alguns efeitos específicos dos herbicidas, a persistência é um dos parâmetros mais importantes a ser conhecido para avaliação de seu impacto ambiental. Os ensaios biológicos, mediante utilização de plantas e de microorganismos, são métodos simples, econômicos e eficientes para avaliação da persistência de herbicidas em solos, desde que sejam tomadas certas precauções (Santelman et al., 1971). Estudos realizados com o linuron em latossolo vermelho álico sugerem que esse herbicida apresentou baixa atividade biológica no solo (Sena Filho \& Machado Neto, 1985). As dinitroanilinas, em geral, não são prejudiciais às culturas subseqüentes, desde que empregadas nas doses recomendadas (Romanowski \& Libik, 1978). Este trabalho tem como objetivo determinar, em condiçōes de campo, a persistência do linuron e da orizalina em soja cultivada em latossolo roxo, na presença de Bradyrhizobium japonicum.

\section{MATERIAL E METODOS}

O experimento foi realizado na Estação Experimental de Ribeirão Preto (SP) durante dois anos consecutivos, em condiçōes usuais de cultivo de soja, Glycine max (L.) Merrill, 'IAC-11'. O plantio foi efetuado no primeiro ano, em 2/12/87 e no segundo, em 25/11/88. O solo, um latossolo roxo, cuja análise química se encontra no quadro 1 , foi corrigido e adubado de acordo com as instruçooes de Mascarenhas (1985).

$O$ experimento foi instalado em parcelas subdivididas com os tratamentos das parcelas principais dispostos em blocos ao acaso, com quatro repetiçöes. As parcelas eram compostas de onze linhas de $5 \mathrm{~m}$ de comprimento, espaçadas de $0,60 \mathrm{~m}$, com os seguintes tratamentos: linuron nas doses $1,00 \mathrm{e}$ $2,00 \mathrm{~kg} / \mathrm{ha}$; orizalina nas doses 1,875 e $3,75 \mathrm{~kg} / \mathrm{ha}$ e duas testemunhas sem herbicidas. As sementes de todas as parcelas tratadas com herbicidas e de uma das testemunhas foram inoculadas com Bradyrhizobium japonicum, estirpe SMS-443 (= 29W), na dose de $8 \mathrm{~g}$ de inoculante turfoso por quilograma de semente.

Os herbicidas foram aplicados em pré-emergência com pulverizador costal, dotado de barra com dois bicos de jato plano da série 80.03 , com consumo de calda correspondente a $250 \mathrm{l} / \mathrm{ha}$.

As subparcelas eram referentes às diferentes épocas de amostragem feitas nas entrelinhas das parcelas para a análise da persistência dos herbicidas.

\section{Teste preliminar para sensibilidade dos bioensaios para análise dos herbicidas}

Uma amostra de solo composta da área experimental foi coletada antes da instalação do experimento para se comparar a sensibilidade dos métodos de Santelman (1977) e Parker (1966), para análise da persistência do linuron e da orizalina.

Como plantas-testes para linuron e orizalina foram usados, respectivamente, pepino (Cucumis 
sativus L., 'Sprint') e sorgo (Sorghum bicolor (L.) Moench, 'DK-867'). As subamostras de solo foram contaminadas com ambos os herbicidas nas concentraçōes de $0,0,02,0,05,0,1,0,2,0,5,1,2$ e $5 \mathrm{mg} / \mathrm{kg}$.

\section{Análise de persistência}

A partir da aplicação dos herbicidas, realizaram-se, para análise da persistência, onze coletas de amostras de solo em intervalos de 14 dias até 126 dias e mais uma aos 160 dias da primeira aplicação. No segundo ano, efetuaram-se as amostragens aos $0,28,56,70,84,105,145$ e 160 dias da primeira aplicação, para o linuron, e ainda aos 390 dias, para a orizalina. Como nessa última época de amostragem não havia mais plantas nas parcelas, coletaram-se dez subamostras de cada parcela. As amostras foram misturadas, destorroadas e peneiradas em peneiras de $2 \mathrm{~mm}$ antes de utilizadas para o teste.

Os métodos de Santelman et al. (1971) e de Parker (1966) foram aplicados na forma descrita por Novo et al. (1991), sendo, no método de Santelman, usadas duas plantas de pepino ou três de sorgo, por recipiente, e as determinaçōes feitas aos 24 dias do plantio.

A relação (R) entre doses de herbicidas e redução do sistema radicular ou da parte aérea das plantas, expressa em porcentagem, foi estudada com a função logística:

$$
\mathbf{R}=\alpha /[1+\exp \cdot(\beta+\delta \mathbf{x})]
$$

onde:

$\mathbf{R}=$ redução do crescimento do sistema radicular ou da parte aérea calculada por

$$
R=\frac{(L o-L t)}{L o} \times 100,
$$

onde:

Lo = média do peso da matéria fresca da parte aérea ou média do comprimento do sistema radicular na testemunha inoculada;

$L t=$ média do peso da matéria fresca da parte aérea ou média do comprimento do sistema radicular para os tratamentos com herbicidas.

$x$ = logaritmo da concentração do herbicida em $\mathrm{mg} / \mathrm{kg}$.

A concentração de herbicida que causava $50 \%$ da redução no crescimento, $\mathrm{RC}_{50}$, foi estimada pela equação:

$$
\mathrm{RC}_{50}=\frac{-\beta}{\delta}
$$

\section{RESULTADOS E DISCUSSÃO}

Os resultados dos testes preliminares para a comparação dos métodos para análise da persistência de orizalina e linuron podem ser observados nas figuras 1 e 2 respectivamente.

Quadro 1. Análise química de amostras de solo coletadas em 1986 e 1987 no local do ensaio, em Ribeirão Preto (SP)

$\begin{array}{lllllllllll}\text { Ano } & \mathrm{P} & \text { M.O. } & \mathrm{pH} \mathrm{CaCl}_{2} & \mathrm{~K}^{+} & \mathrm{Ca}^{2+} & \mathrm{Mg}^{2}+\left(\mathrm{H}+\mathrm{Al}^{3+}\right) & \mathrm{S} & \mathrm{T} & \mathrm{V}\end{array}$

\begin{tabular}{cccccccccccc} 
& & & & & \multicolumn{9}{c}{ m $/ 100 \mathrm{~cm}^{3}$} & $\%$ & & \multicolumn{3}{c}{ meq/100ml T.F.S.A. } & & & $\%$ \\
1986 & 58,0 & 3,6 & 4,9 & 0,52 & 3,2 & 1,2 & 4,3 & 4,9 & 9,2 & 52,0 \\
1987 & 41,0 & 3,6 & 4,9 & 0,29 & 2,9 & 0,9 & 4,0 & 4,1 & 8,1 & 51,0 \\
\hline
\end{tabular}


O método de Parker - Figura 1 - indica que, para a orizalina, a redução do crescimento da raiz do sorgo foi acentuada no intervalo das doses $\mathbf{0 , 0 5}$ e $1,00 \mathrm{mg} / \mathrm{kg}$ e que, com o aumento da concentração do herbicida, essa reduçāo tende para um assíntota; assim, para doses acima de $1,00 \mathrm{mg} / \mathrm{kg}$, a redução é um pouco superior a $80 \%$. Observa-se, ainda, que, por esse método, foi necessária uma concentração de $0,302 \mathrm{mg} / \mathrm{kg}$ de orizalina para causar redução de $50 \%$ no crescimento da raiz do sorgo, enquanto, pelo de Santelman, para uma redução equivalente na parte aérea, foi necessária a concentração de $0,729 \mathrm{mg} / \mathrm{kg}$ do mesmo produto.

Pela observação das plântulas de sorgo, notou-se que houve inibição tanto da raiz principal como das secundárias, além de engrossamento da raiz principal. Não houve diferenças nos sintomas em virtude de diferentes concentraçōes dos herbicidas. Ashton \& Crafts (1973) relataram que as dinitroanilinas, como é o caso da orizalina, inibem tanto o crescimento da raiz como da parte aérea.

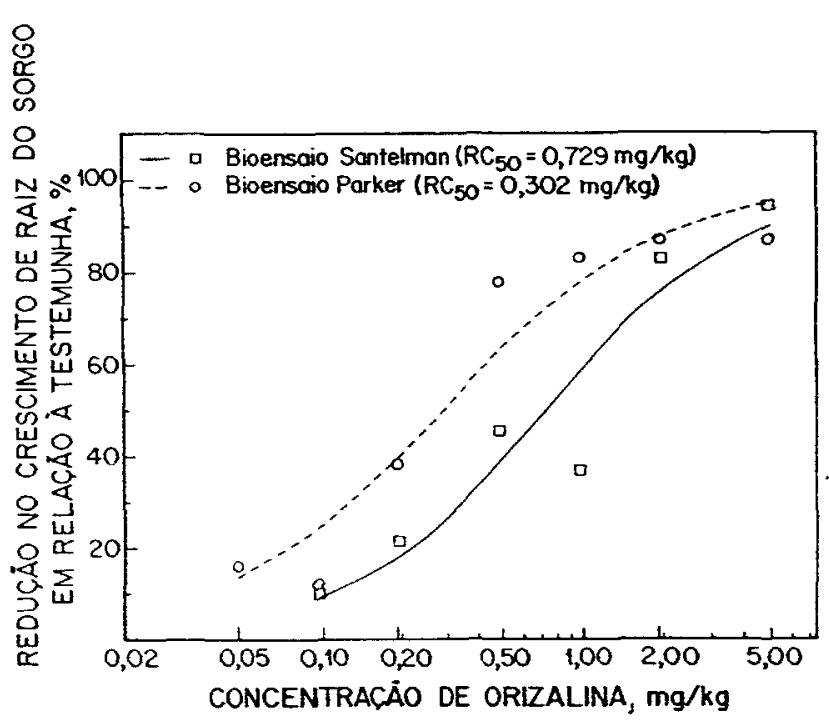

Figura 1. Teste preliminar para a sensibilidade dos métodos de Parker e Santelman a diferentes concentraçöes de orizalina, usando sorgo como planta-teste.
Parker (1965) observou que a aplicação de orizalina resultou em respostas muito pequenas no crescimento da parte aérea (mediçōes do caulículo); por essa razão, essa avaliação nāo foi considerada neste ensaio. Durante o crescimento da parte aérea, há pouco movimento interno do herbicida na plântula e qualquer redução de crescimento nesse estádio inicial será conseqüência de absorção via não radicular.

Apesar de o método de Parker ter sido bom indicador da atuação da orizalina, nāo foram obtidas informações sobre a absorção radicular do produto em vista da considerável redução no crescimento da planta.

O linuron - Figura 2 - teve pouco efeito no crescimento da raiz, o que confirma as observaçōes de Parker (1966). Mesmo na concentração de 5 ppm, a redução no crescimento da raiz foi pequena, correspondendo a $32 \%$ em relação à testemunha. O método de Santelman mostrou maior sensibilidade, sendo a RC50 observada de $0,675 \mathrm{mg} / \mathrm{kg}$.
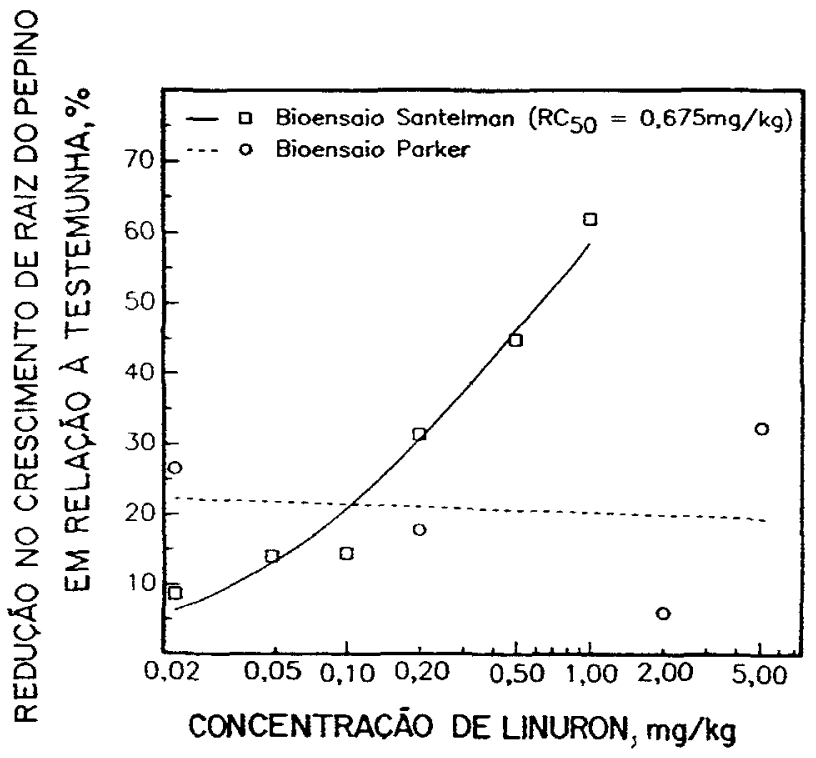

Figura 2. Teste preliminar para a sensibilidade dos métodos de Parker e Santelman a diferentes concentraçōes de linuron, usando pepino como planta-teste. 
Sena Filho \& Machado Neto (1985) observaram que a atividade do linuron é baixa em solo de cerrado, pela baixa solubilização do produto na solução do solo (75 ppm de linuron em água a $\left.25^{\circ} \mathrm{C}\right)$.

É possível que o sorgo seja mais sensível à orizalina que o pepino ao linuron, em vista da própria anatomia da planta. $O$ sorgo, como as demais monocotiledôneas, tem o meristema apical e outros tecidos não radiculares mantidos no solo antes que as primeiras folhas se expandam. Zonas sensíveis ao herbicida ficam em contato com o solo contaminado por um período considerável. Nas dicotiledôneas, caso do pepino, o meristema apical emerge do solo na mesma época que o cotilédone, sendo menor o contato com o solo. Essa diferença na forma do desenvolvimento inicial pode explicar a maior sensibilidade do sorgo que do pepino no método de Parker.
Tendo em vista os resultados de Horowitz (1969), sugerindo que a perda da atividade da trifluralina (também uma dinitroanilina) é mais rápida em amostras contaminadas no laboratório do que nas coletadas onde o produto foi aplicado no campo, para avaliação da persistência da orizalina, no primeiro ano, empregaram-se ambos os métodos. Para a análise de persistência do linuron, somente o método de Santelman.

Em condições de campo, o método de Parker e o de Santelman para orizalina - Figura 3A e B - nas amostras colhidas no primeiro ano de cultivo, mostraram o mesmo resultado do teste preliminar, ou seja, as raízes foram mais sensíveis que a matéria fresca da parte aérea praticamente em todas as amostras. No segundo ano de cultivo, a persistência da orizalina foi determinada somente pelo método de Parker.
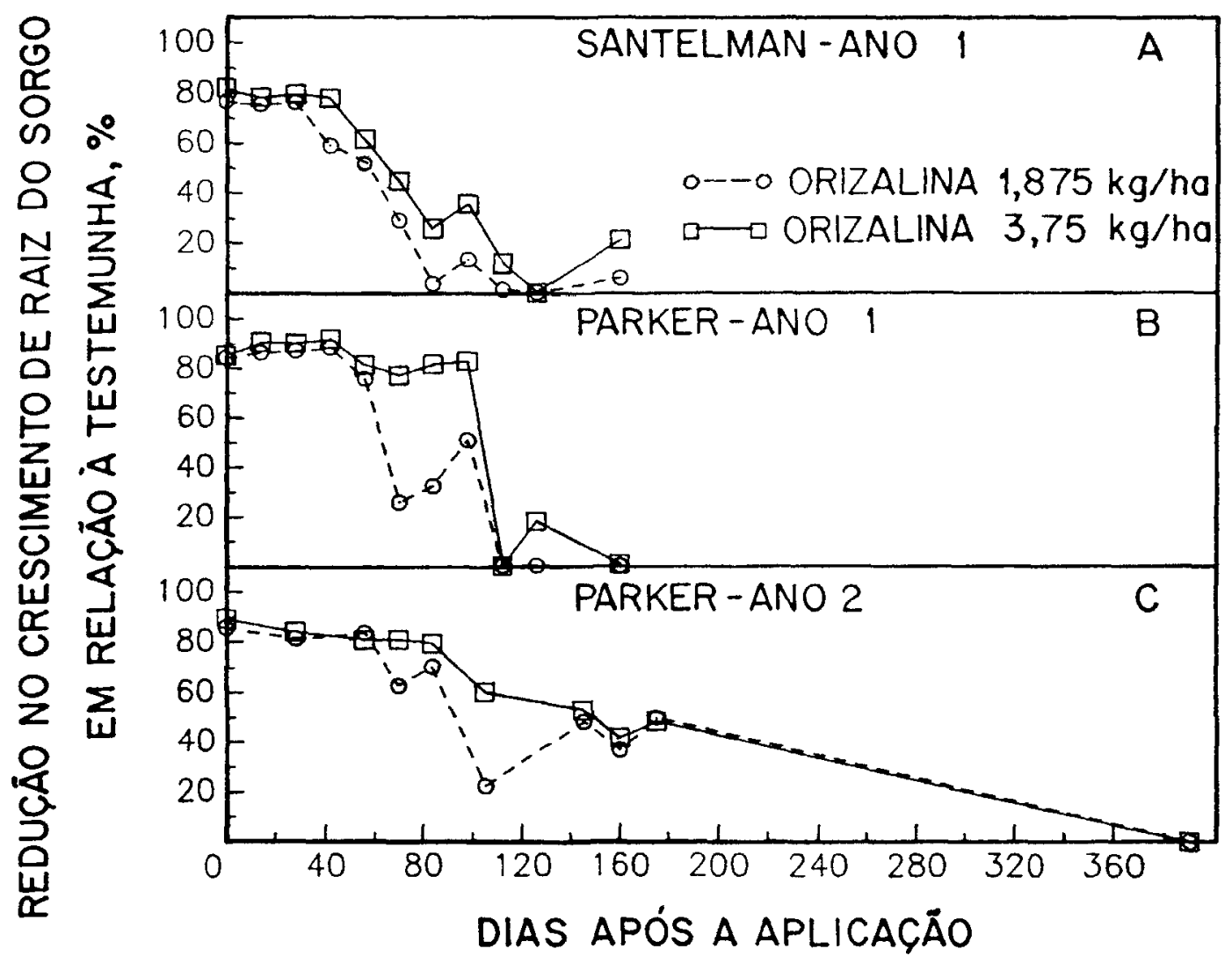

Figura 3. Persistência de duas doses de orizalina, em latossolo roxo cultivado com soja em Ribeirăo Preto (SP), determinada por bioensaio. Os dados são médias de quatro repetiçôes utilizando sorgo como planta-teste em solo amostrado em diversos períodos após a aplicação do produto. 
O desenvolvimento da planta-teste em solo tratado com as duas doses de orizalina nas diversas épocas de amostragem de solo, observadas no primeiro e no segundo ano, é apresentado na figura 3B e C.

Observa-se que, no primeiro ano, a perda da atividade da orizalina foi lenta até os 56 e 84 dias para as doses de 1,875 e $3,75 \mathrm{~kg} / \mathrm{ha}$ respectivamente. No segundo ano, as doses $1,875 \mathrm{~kg} / \mathrm{ha}$ aos 84 dias e $3,75 \mathrm{~kg} / \mathrm{ha}$ aos 145 dias ainda reduziam o crescimento da planta-teste em mais de $50 \%$.

No primeiro ano, na amostragem realizada aos 112 dias, o desenvolvimento das raízes de sorgo em solo tratado tanto com $1,875 \mathrm{~kg} / \mathrm{ha}$ como com 3,75 $\mathrm{kg} / \mathrm{ha}$ de orizalina não apresentou diferença com relaçāo à testemunha. No segundo ano, o mesmo só ocorreu aos 390 dias da aplicação.

Nota-se que a orizalina, nos dois anos de cultivo, mostrou um padrão inicial lento de desaparecimento, seguido de aumento na taxa de degradação, o que sugere uma fase de espera (lag fase). Parka \& Tepe (1969) observaram que dinitroanilinas não são translocadas para camadas de solo mais profundas e se movimentam muito pouco no solo; mesmo assim, não há acúmulo no solo após repetidos tratamentos anuais. No Brasil, essa observação foi comprovada por Meneghel et al. (1980), que relataram a permanência no solo, após a colheita da soja, de 3,7 a $9,3 \%$ da dose aplicada.
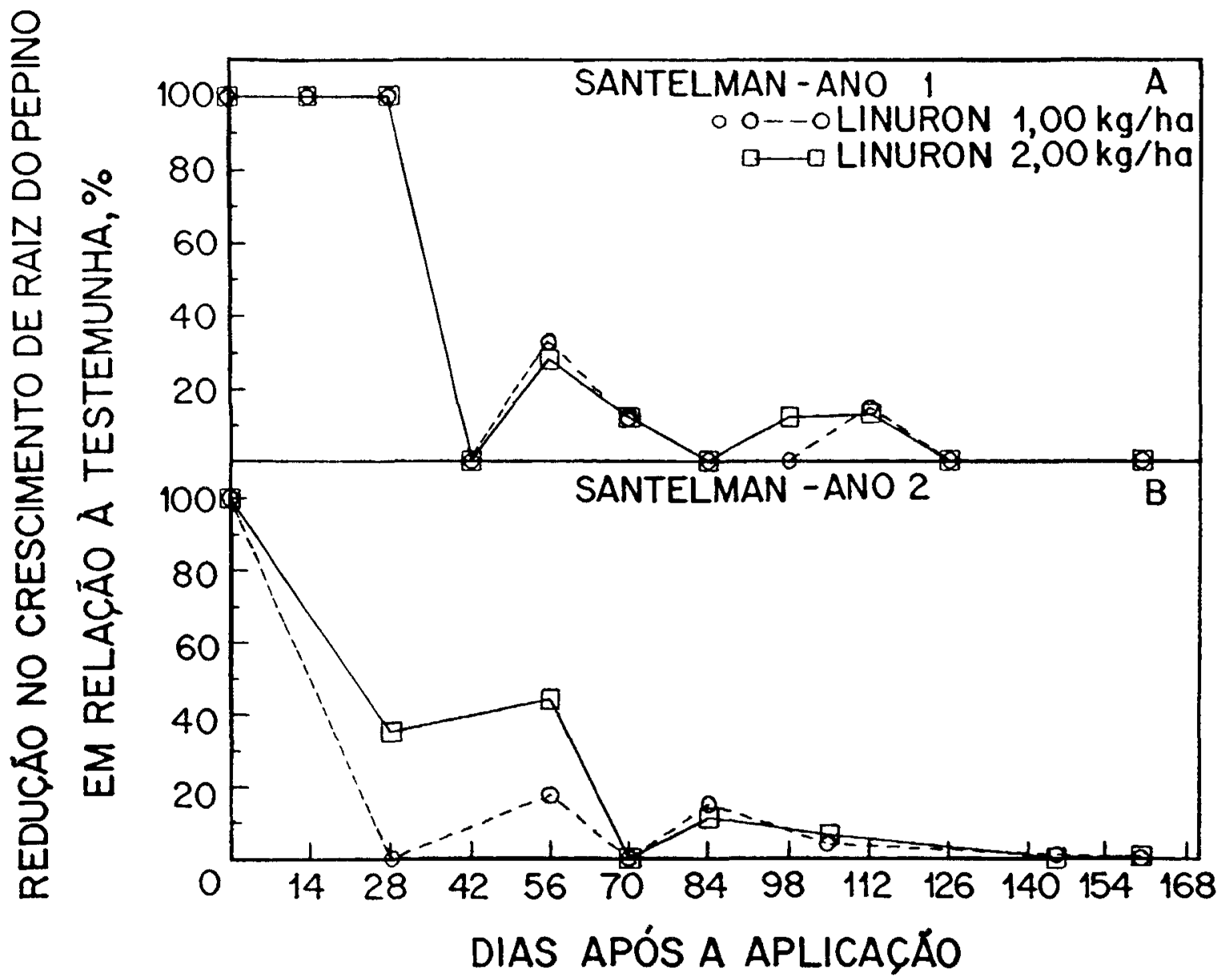

Figura 4. Persisténcia de duas doses de linuron, em latossolo roxo cultivado com soja em Ribeirāo Preto (SP), determinado por bioensaio. Os dados săo médias de quatro repetiçōes, utilizando pepino como planta-teste em solo amostrado em diversos períodos após a aplicação do produto. 
Golab et al. (1975) determinaram que a meia-vida da orizalina sob condições aeróbicas e não aeróbicas a $30^{\circ} \mathrm{C}$ são, respectivamente, 1,4 e 0,34 mês. Isso sugere maior degradação não biológica. Provavelmente as perdas desse produto se devam à fotodecomposição, volatilização e degradação química nāo biológica, conforme demonstraram Probst et al. (1967); Probst \& Tepe (1969) e Messersmith et al. (1971).

Analisando-se os dados de persistência do linuron - Figura 4 - nas amostragens realizadas logo após a aplicação, observa-se que a fitotoxicidade inicial desse produto é bastante elevada: no primeiro ano, até os 28 dias, a quantia de herbicida do solo causava morte na planta-teste. A partir dessa data, porém, a degradação do linuron foi relativamente rápida, mesmo na dose de $2,00 \mathrm{~kg} / \mathrm{ha}$, não havendo, aos 42 dias, sintomas de fitotoxicidade devidos a nenhuma dose.

No segundo ano - Figura 4B - a degradação do produto, aplicado na dose de $1,00 \mathrm{~kg} / \mathrm{ha}$, foi mais rápida, nāo havendo sintoma de fitotoxicidade aos 28 dias; a dose de $2,00 \mathrm{~kg} / \mathrm{ha}$, porém, ainda persistia, reduzindo o crescimento da planta-teste em $64,9 \%$. Somente na amostragem realizada aos 70 dias é que essa dose não mais causou fitotoxicidade. A partir de então, houve sintomas ocasionais na planta-teste.

Fryer \& Kirkland (1970) observaram que a maior quantidade de linuron é encontrada nos primeiros 5cm do solo; portanto, a perda da atividade do produto não pode ser devida à percolação à camada mais profunda. A rápida degradação, segundo Walker (1978), também não se deve à temperatura. Hill et al. (1955) relatam que a rápida degradaçāo do linuron deve estar relacionada à sua decomposição microbiana, embora Lode (1967) tenha comprovado que, após a aplicação, o produto ocasiona diminuição na população microbiana do solo, que posteriormente é restabelecida.

Hill et al. (1955) citam microorganismos de solo capazes de utilizar herbicidas derivados da uréia como fontes de nitrogênio para suas necessidades energéticas. Weinberger \& Bollag (1972) apontam o fungo do solo Rhizoctonia solani como um dos organismos que degradam linuron e outras uréias.
Segundo Kaufmann et al. (1985) e Fryer \& Kirkland (1970), sob certas condiçōes, quando repetidas aplicações de pesticidas são feitas no mesmo local, a persistência e a eficiência do produto diminuem, havendo ausência de uma lag fase mais longa no processo de degradação de alguns pesticidas de solo.

\section{CONCLUSŌES}

1. Os testes preliminares para a comparação de métodos para análise da persistência dos herbicidas mostraram que, para o linuron, o método de Santelman foi mais sensível e para a orizalina, o de Parker.

2. A perda de atividade da orizalina foi lenta, com atividade, no primeiro ano de aplicação, até os 112 dias, não apresentando, no segundo, fitotoxicidade na planta-teste aos $\mathbf{3 9 0}$ dias da aplicação.

3. A degradação do linuron foi relativamente rápida, não havendo, no primeiro ano, sintomas de fitotoxicidade aos 42 dias após a aplicação para ambas as doses, enquanto, no segundo ano, a ausência de fitotoxicidade ocorreu aos 42 e 70 dias, respectivamente, para as doses 1,00 e $2,00 \mathrm{~kg} / \mathrm{ha}$.

\section{REFERENCIAS BIBLIOGRÁFICAS}

ASHTON, F.M. \& CRAFTS, A.S. Mode of action of herbicides. New York, Willey-Interscience, 1973. 504p.

FRYER, J.D. \& KIRKLAND, K. Field experiments to investigate long-term effects of repeated applications of MCPA, tri-allate, simazine and linuron: report after 6 years. Weed Research, Oxford, 10(2):133-158, 1970.

GOL AB, T.C.E.; BISHOP, A.I.D.; DONOHO, A.L.; MANTHEY, J.A. \& ZORNES, LL. Behaviour, of 14c-oryzalin in soils and plants. Pesticide Biochemistry and Physiology, San Diego, 5(2):196-204, 1975.

HERTWIG, K. von, coord. Manual de herbicidas destolhantes, dessecantes, fitorreguladores e bio-estimulantes. 2.ed. São Paulo, Agronômica Ceres, 1983. 670p.

HILL, G.D.; MCGAHEN, J.W.; BAKER, H.M.; FINNERTY, D.W. \& BINGEMAN, C.W. The fate of substituted urea herbicides in agricultural soils. Agronomy Journal, Madison, 47(2):93$-104,1955$. 
HOROWIIZ, M. Evaluation of herbicide persistence in soil. Weed Research, Oxford, 9(4):314-321, 1969.

KAUFMAN, D.D.; HORNG, L.C. \& AHARONSON, N. Loss of efficacy of pesticides due to rapid biodegradation by soil microorganisms. In: ANNUAL CORN \& SORGHUM INDUSTRY RESEARCH CONFERENCE, 40., Chicago, 1985. Proceedings. Washington, D.C., American Seed Trade Association, 1985. p.175-191. (Publication, 40)

LODE, O. Decomposition of linuron in different soils. Weed Reseanch, Oxford, 7(2):185-190, 1967.

MASCARENHAS, H.A.A. Soja. In: RAJ, B. van; SILVA, N.M. da; BATAGLIA, O.C.; QUAGGIO, J.A.; HIROCE, R.; CANTARELLA, H.; BELLINAZZI JÚNIOR, R; DECHEN, A.R \& TRANI, P.E. Recomendaçöes de adubaçäo e calagem para o Estado de São Paulo. Campinas, Instituto Agronômico, 1985. p.20. (Boletim técnico, 100)

MENEGHEL, D.; HING, M.W.; CAMPOS, R.B.; MACHADO, P.R. \& ROCHA, C.L. Orizalina: culturas sequenciais à soja e resíduos no solo. In: CONGRESSO BRASILEIRO DE HERBICIDAS E ERVAS DANINHAS, 13., Ilhéus/Itabuna, 1980. Resumos. Ilhéus/Itabuna, Centro de Pesquisa do Cacau, 1980. p.100.

MESSERSMITH, C.G.; BURNSIDE, O.C. \& LAVY, T.L. Biological and non-biological dissipation of trifluralin from soil. Weed Science, Champaign, 19(3):285-290, 1971.

NOVO, M. do C. de S.S.; LOPES, E.S. \& ORTOLAN, M.C.A. Levantamento da nodulaçăo, persistência de herbicidas e isolamento de Bradyrhizobium sp. de áreas com rotaçāo cana-de-açúcar/amendoim. Revista de Microbiologia, São Paulo, 22(1):60-65, 1991.

PARKA, S.J. \& TEPE, J.B. The disappearance of trifluralin from field soils. Weed Science, Champaign, 17(1):119-122, 1969.
PARKER, C. A rapid bio-assay method for the detection of herbicides which inhibit photossynthesis. Weed Research, Oxford, S(1):181-184, 1965.

PARKER, C. The importance of shoot entry in the action of herbicides applied to the soil. Weeds, Champaign, 14(2):117-121, 1966.

PROBST, G.W.; GOLAB, T.; HERBERG, RJ.; HOLZER, F.J.; PARKA, SJ.; SCHANS, C. van der \& TEPE, J.B. Fate of trifluralin in soils and plants. Journal of Agricultural and Food Chemistry, Washington, D.C.; 15(4):592-599, 1967.

PROBST, G.W. \& TEPE, J.B. Trifluratin and related compounds. In: KEARNEY, P.C. \& KAUFMAN, D.D., eds. Degradation of herbicides. New York, Marcel Deckker, 1969. p.255-282.

ROMANOWSKI, R.R. \& LIBIK, A.W. Soil persistence of isopropalin, nitralin, and trifluralin. Weed Science, Champaign, 26(3):258-261, 1978.

SANTELMAN, P.W. Herbicide bioassay, In: SOUTHERN WEED SCIENCE SOCIETY. Research methods in weed science. 2.ed. Georgia, 1977. p.79-87.

SANTELMAN, P.W.; WEBER, J.B. \& WIESE, A.F. A study of soil bioassay technique using prometryne. Weed Sclence, Champaign, 19(2):170-174, 1971.

SENA FILHO, A. \& MACHADO NETO, J.G. Atividade biológica de metribuzin e linuron em duas unidades de um latossolo vermelho originalmente sob vegetação de cerrado. Pesquisa Agropecuária Brasileira, Brasilia, 20(5):545-550, 1985.

WALKER, A. Simulation of the persistence of eight soil-applied herbicides. Weed Research, Oxford, 18(5):305-313, 1978.

WEINBERGER, M. \& BOLLAG, J.M. Degradation of chlorbromuron and related compounds by the fungus Rhizoctonia solani. Applied Microbiology, Baltimore, 24(5):750-754, 1972. 